\title{
Necessary Condition for Observer-Based Chaos Synchronization
}

\author{
Ömer Morgül* \\ Department of Electrical and Electronics Engineering, Bilkent University, 06533, Bilkent, Ankara, Turkey
}

(Received 5 June 1998)

\begin{abstract}
We consider observer-based synchronization of chaotic systems. In this scheme, for a given chaotic drive system, response system is chosen in observer form. We show by examples that many response systems proposed in the literature are of this form. We give a necessary condition for synchronization and a selection criterion for appropriate synchronization signal in this case. We apply this idea to synchronization of well-known hyperchaotic Rössler system. [S0031-9007(98)08057-0]
\end{abstract}

PACS numbers: 05.45.Xt, 43.72.+q

Although the idea of synchronization of chaotic systems may seem impossible to achieve, it was shown in $[1,2]$ that for certain chaotic systems such synchronization is possible. This subject then received a great deal of attention among scientists in many fields; see, e.g., [37]. Such synchronized chaotic systems usually consist of two parts: a generator of chaotic signals (drive system) and a receiver (response) system. The response system is usually a duplicate of a part (or whole) of the drive system and some signals generated in the drive system are used as synchronization signals in the response system. Although many synchronization schemes are proposed in the literature, most of these schemes do not give a systematic procedure to determine the response system and the synchronization signal. Recently an observer-based synchronization scheme has been proposed in [8,9], which gives a systematic design procedure to guarantee synchronization for certain chaotic systems.

In this Letter we will consider the observer-based synchronization scheme. First we will show that many synchronization schemes proposed in the literature are observer based. Then we will consider two problems concerning observer-based synchronization. Namely, we will give a necessary condition for synchronization and a criterion for the selection of an appropriate synchronization signal among various candidates satisfying the necessary condition.

Let a chaotic (drive) system be given as

$$
\dot{u}=f(u), \quad s=h(u),
$$

where $u \in \mathbf{R}^{n}, f: \mathbf{R}^{n} \rightarrow \mathbf{R}^{n}$ and $h: \mathbf{R}^{n} \rightarrow \mathbf{R}^{m}$ are differentiable functions, and $s$ is the synchronization signal to be sent to the response system. Later, for simplicity, we will choose $m=1$, i.e., a scalar synchronization signal. An observer for (1) is another system of the form

$$
\dot{v}=g(v, s), \quad u_{r}=k(v, s),
$$

where $v \in \mathbf{R}^{l}, \quad g: \mathbf{R}^{l} \times \mathbf{R}^{m} \rightarrow \mathbf{R}^{l}$ and $k: \mathbf{R}^{l} \times$ $\mathbf{R}^{m} \rightarrow \mathbf{R}^{n}$ are differentiable functions. Let the error signal be defined as $e=u-u_{r}$. The system (2) is called a local observer for (1) if $e(t) \rightarrow 0$ as $t \rightarrow \infty$ for all sufficiently small $e(0)$, i.e., when $\|e(0)\| \leq \gamma$ for some $\gamma>0$. If $\gamma=\infty$, then the observer is global. Also, for the cases $l<n, l=n$, and $l>n$, corresponding observers are called reduced, full, and expanded order observers, respectively. We note that the classification of observers based on the order is not important for our discussion here. But we included it to emphasize that, although many schemes proposed in the literature may seem different mainly because of the order of the proposed response systems, they could still be considered as special cases of the observer form given by (2). For more details on nonlinear observers, see [10], and for linear observers, see [11].

Next, we will show that some well-known synchronization schemes proposed in the literature are actually observer based. First we consider the well-known PecoraCarroll scheme proposed in [1]. Consider (1) and assume that we can divide the state space into two parts as $u=\left(\begin{array}{ll}u_{1} & u_{2}\end{array}\right)^{T}$ with $u_{1} \in \mathbf{R}^{m}, u_{2} \in \mathbf{R}^{n-m}$, and consider the following system:

$$
\begin{gathered}
\dot{u}_{1}=f_{1}\left(u_{1}, u_{2}\right), \quad \dot{u}_{2}=f_{2}\left(u_{1}, u_{2}\right), \\
\dot{v}=f_{2}\left(u_{1}, v\right),
\end{gathered}
$$

where the superscript $T$ denotes the transpose. Here, $f_{1}$ and $f_{2}$ are appropriate partitionings of $f$ in (1) and $v \in$ $\mathbf{R}^{n-m}$. In this scheme, the first two equations represent the drive system, the last equation represents the response system, and $u_{1}$ is the synchronization signal. Assume that the subsystems $u_{2}$ and $v$ synchronize, i.e., $u_{2} \rightarrow v$ as $t \rightarrow \infty$. According to the observer scheme, here we have $s=u_{1}=C u$ with $C=(I 0)$, with $I \in \mathbf{R}^{m \times m}$, $g(v, s)=f_{2}(s, v)$, and $u_{r}=(s v)^{T}$. Since $m<n$, this scheme is actually a reduced order observer scheme. Hence, the Pecora-Carroll scheme proposed in [1] is observer based.

Next, we will give some specific examples. Consider the Lorenz system,

$$
\begin{gathered}
\dot{x}=\beta(y-x), \quad \dot{y}=r x-y-x z, \\
\dot{z}=-b z+x y .
\end{gathered}
$$

In [2], by using $s=y$ as the synchronization signal, the following response system is proposed:

$$
\dot{x}_{r}=\beta\left(s-x_{r}\right), \quad \dot{z}_{r}=-b z_{r}+s x_{r} .
$$


For $u=\left(\begin{array}{lll}x & y & z\end{array}\right)^{T} \in \mathbf{R}^{3}$ and $s=y=C u$ with $C=$ $\left(\begin{array}{lll}0 & 1 & 0\end{array}\right)$, the drive system (4) is in the form (1), and with $v=\left(x_{r} z_{r}\right)^{T} \in \mathbf{R}^{2}, u_{r}=\left(\begin{array}{lll}x_{r} & s & z_{r}\end{array}\right)^{T}$, the response system (5) is in the form (2). In this case, $l=2$ and $n=3$, hence (5) is a reduced order observer.

For (4), by using $s=x$, the following response system is proposed in [3]:

$$
\begin{gathered}
\dot{x}_{r}=\beta\left(y_{r}-x_{r}\right), \quad \dot{y}_{r}=r s-y_{r}-s z_{r}, \\
\dot{z}_{r}=-b z_{r}+s y_{r} .
\end{gathered}
$$

Here we have $s=x=C u$ with $C=\left(\begin{array}{lll}1 & 0 & 0\end{array}\right), v=u_{r}=$ $\left(\begin{array}{lll}x_{r} & y_{r} & z_{r}\end{array}\right)^{T} \in \mathbf{R}^{3}$. In this case, $l=n=3$, hence the proposed structure is a full order observer.

In [5-7], the following hyperchaotic Rössler system is considered (see also [12]),

$$
\begin{array}{ll}
\dot{x}_{1}=-x_{2}-x_{3}, & \dot{x}_{2}=x_{1}+0.25 x_{2}+x_{4}, \\
\dot{x}_{3}=3+x_{1} x_{3}, & \dot{x}_{4}=-0.5 x_{3}+0.05 x_{4} .
\end{array}
$$

In [5], by using $s=\sin \theta x_{1}+\cos \theta x_{3}$ as the synchronization signal, where $\theta$ is a constant to be determined, a full order observer similar to the one considered in $[8,9]$ is used. In [6], by using a nonlinear function $h$, a full order observer is proposed. In [7], two types of response systems are proposed. The first one of these is a full order observer, and for the second one the following response system is proposed:

$$
\begin{gathered}
\dot{x}_{1 r}=-x_{2 r}-x_{3 r}, \quad \dot{x}_{2 r}=x_{1 r}+0.25 x_{2 r}+(\mu+1) x_{4 r}, \\
\dot{x}_{3 r}=3+x_{1 r} x_{3 r}, \\
\dot{x}_{4 r}=-0.5 x_{3 r}+(\mu+0.05) x_{4 r} \\
\dot{\mu}=-\alpha\left(s_{r}-s\right)-\delta \mu
\end{gathered}
$$

where $s=\cos \theta x_{2}+\sin \theta x_{4}, s_{r}$ is defined similarly, $\theta$ is a constant to be chosen, and $\alpha>0, \delta>0$ are appropriate constants. Here we have $u=\left(x_{1} \cdots x_{4}\right)^{T} \in \mathbf{R}^{4}, s=$ $C u$ with $C=(0 \cos \theta 0 \sin \theta), v=\left(x_{1 r} \cdots x_{4 r} \mu\right)^{T} \in$ $\mathbf{R}^{5}, u_{r}=\left(x_{1 r} \cdots x_{4 r}\right)^{T}$. In this case we have $l=5$, $n=4$; hence (8) is an expanded order observer for (7).

The examples given above show that the observerbased synchronization is a very common scheme. Next, we will consider the following problems concerning observer-based synchronization:

Problem 1: Given the drive system dynamics [i.e., $f$ in (1)], to determine appropriate synchronization signals [i.e., $h$ in (1)], which may (or conversely, may not) lead to synchronization.

Problem 2: To develop a selection procedure among the various synchronization signal candidates.

In most of the examples given in the literature, problem 1 is solved by a trial and error procedure, and an appropriate Lyapunov exponent of the error dynamics is used as a selection criterion for problem 2 . We will give a necessary condition for problem 1 which is based on linearization and propose a novel selection criterion for problem 2 which is based on the singular values of the linearized system.
Our approach is based on the concepts of detectability and observability, which are frequently used in linear system theory; see, e.g., [11,13]. Let $A \in \mathbf{R}^{n \times n}$ and $C \in \mathbf{R}^{m \times n}$ be given. The pair $(C, A)$ is called detectable if there exists a matrix $K \in \mathbf{R}^{n \times m}$ such that the matrix $A-K C$ is stable. The pair $A-K C$ is called observable if for any set of (real or complex) numbers $\lambda_{1}, \ldots, \lambda_{n}$ (not necessarily distinct but closed under complex conjugation), there exists a matrix $K$ such that the eigenvalues of $A-K C$ are precisely the given numbers. Note that observability implies detectability, but the converse is not necessarily true.

To motivate our analysis, consider the well-known Pecora-Carroll scheme given in (3). Consider the following linearization of (3):

$$
\begin{gathered}
\dot{u}_{1}=A_{11} u_{1}+A_{12} u_{2}+o_{1}\left(u_{1}, u_{2}\right), \\
\dot{u}_{2}=A_{21} u_{1}+A_{22} u_{2}+o_{2}\left(u_{1}, u_{2}\right), \\
\dot{v}=A_{21} u_{1}+A_{22} v+o_{2}\left(u_{1}, v\right),
\end{gathered}
$$

where, for $i, j=1,2, A_{i j}$ are appropriate matrices and $o_{i}$ represent the remaining nonlinear terms. If we define the error as $e=u_{2}-v$, then the associated error dynamics is given as $\dot{e}=A_{22} e+o_{2}\left(u_{1}, u_{2}\right)-o_{2}\left(u_{1}, v\right)$. Hence, if synchronization occurs, i.e., $e \rightarrow 0$ as $t \rightarrow \infty$, then $A_{22}$ must be stable. To see the relation of this result with detectability, let $A=\left\{A_{i j}\right\}$ denote the block matrix having entries $A_{i j}$ and choose $K=\left(K_{1}^{T} K_{2}^{T}\right)^{T}$ with $K_{1}=$ $A_{11}+I, K_{2}=A_{21}$. Then the eigenvalues of $A-K C$ are precisely -1 and the eigenvalues of $A_{22}$. Hence, if the Pecora-Carroll synchronization scheme is successful, then the linearization is detectable. The scheme proposed in [8] and [9] is based on a special full order observer design and depends on the selection of an appropriate feedback matrix $K$ such that $A-K C$ is stable, hence detectability is also a requirement there. It follows that for the schemes of $[1,8,9]$, detectability is a necessary condition. However, these schemes are proposed for some special systems, and whether such a necessary condition also applies to all kinds of observer-based schemes, irrespective of the order of the observer, remains as an interesting question. The following well-known result from system theory shows that the answer to this question is affirmative under some mild conditions.

Lemma 1: Consider the system given by (1) and the observer given by (2). Assume that all functions are differentiable. Without loss of generality, let $f(0)=0$, $h(0)=0$, and let $A=D f(0), C=D h(0)$, i.e., the Jacobians at $u=0$. If the error dynamics is asymptotically stable at $e=0$ (i.e., synchronization occurs for all sufficiently small $\|e(0)\|)$, then the pair $(C, A)$ is detectable.

Proof: See [10,14,15].

According to Lemma 1 , if the pair $(C, A)$ is not detectable, then synchronization cannot occur when a differentiable observer in the form (2) is used. Hence, the detectability is a necessary condition for synchronization 
when all dynamics differentiable. Therefore, the synchronization signal $s$ should be chosen so that $(C, A)$ is at least detectable. This result gives an answer to problem 1 posed above. The conditions for a pair $(C, A)$ to be detectable can be found in standard textbooks on linear system theory; see, e.g., [11,13]. To apply this idea to some well-known systems, we give such a condition in the sequel.

For a given pair $(C, A)$, we define the observability matrix $Q$ as

$$
Q=\left[C^{T} A^{T} C^{T} A^{2 T} C^{T} \cdots A^{(n-1) T} C^{T}\right]^{T} .
$$

The pair $(C, A)$ is observable if $\operatorname{det} Q \neq 0$. Now assume that $m=1$ (i.e., a scalar synchronization signal) and that $\operatorname{det} Q=0$. Furthermore, assume that the first $p(p<n)$ rows of $Q$ are linearly independent but the first $p+1$ rows are linearly dependent. Let $R \in \mathbf{R}^{(n-p) \times n}$ be an arbitrary matrix such that $P=$ $\left[C^{T} A^{T} C^{T} \cdots A^{(p-1) T} C^{T} R^{T}\right]^{T}$ is nonsingular. In this case, the matrix $\Delta=P A P^{-1}$ has a block-lower triangular form $\Delta=\left[\Delta_{a} \Delta_{b}\right]$ with $\Delta_{a}=\left[\Delta_{11} \Delta_{21}\right]^{T}, \Delta_{b}=$ $\left[\begin{array}{ll}0 & \Delta_{22}\end{array}\right]^{T}$, where $\Delta_{11} \in \mathbf{R}^{p \times p}$. The pair $(C, A)$ is detectable if $\Delta_{22}$ is stable.

Next, we will apply the necessary condition given above to the synchronization of some well-known chaotic systems. First, consider the Lorenz system given by (4), and let $A$ be the linear part when (4) is written as (1). If $s=x$ is used, then we have $C=\left(\begin{array}{lll}1 & 0 & 0\end{array}\right)$, and $\operatorname{det} Q=0$; hence $(C, A)$ is not observable. To test detectability, we note that in this case $p=2$, and by choosing $R=$ $\left(\begin{array}{lll}0 & 0 & 1\end{array}\right)$ and using $\Delta=P A P^{-1}$, we obtain $\Delta_{22}=-b$, which is stable for $b>0$. Hence, synchronization may be achieved with $s=x$. For $s=y$, we have $C=$ $\left(\begin{array}{lll}0 & 1 & 0\end{array}\right)$, and similar calculations show that the pair $(C, A)$ is not observable, but $p=2$ and we have $\Delta_{22}=-b$, which is stable for $b>0$. Hence synchronization may be possible with $s=y$. For $s=z$, we have $C=$ $\left(\begin{array}{lll}0 & 0 & 1\end{array}\right)$, and $(C, A)$ is not observable. We have $p=1$ in this case, and by choosing an appropriate $R$, we obtain the characteristic polynomial for $\Delta_{22}$ as $\operatorname{det}(\lambda I-$ $\left.\Delta_{22}\right)=\lambda^{2}+(\beta+1) \lambda+\beta(1-r)$. Hence for $\beta>$ 0 , we need $r<1$ for detectability. Hence, if $r>$ 1 , synchronization cannot occur with a differentiable observer.

As another example frequently used in the literature, consider the Rössler system given below,

$$
\begin{gathered}
\dot{x}=-y-z, \quad \dot{y}=x+a y, \\
\dot{z}=-c z+z x+b,
\end{gathered}
$$

and let $A$ denote the linear part when (12) is written as (1) with $u=\left(\begin{array}{lll}x & y & z\end{array}\right)^{T}$. If $s=x$ is used, then $\operatorname{det} Q=a+c$, and the synchronization may be possible when $a+c \neq 0$. We note that in this case (exponential) synchronization is guaranteed with the full order observer given in [9]. If $s=y$ is used, then $\operatorname{det} Q=-1$, hence synchronization may be possible. For $s=z$, we have $\operatorname{det} Q=0$, hence $(C, A)$ is not observable. We have $p=1$ in this case, and by choosing an appropriate $R$, we obtain the characteristic polynomial of $\Delta_{22}$ as $\operatorname{det}\left(\lambda I-\Delta_{22}\right)=\lambda^{2}-a \lambda+1$, which is unstable for $a>0$. Hence, in this case, synchronization is not possible by using differentiable observers. At this point, we note that in [16], a different synchronization scheme based on impulsive coupling is given (i.e., a synchronization signal is used only at certain instances), and it was noted that synchronization is possible with $s=z$. This result does not contradict our conclusion, since in this case the response system contains impulsive terms; hence it is not differentiable.

Next, we consider problem 2. Let $A$ and $C$ denote the linear parts of $f$ and $h$, respectively. We will assume that $(C, A)$ is observable, since this condition is sufficient in many cases. Suppose that for a given $A$, the candidates for $C$ satisfying the observability condition are parametrized, e.g., by $\theta \in \Omega \subset \mathbf{R}^{q}$. Then the problem is to find $\theta_{*} \in \Omega$ which yields better synchronization properties. One approach may be to calculate appropriate Lyapunov exponents of the error dynamics and choose $\theta_{*}$ accordingly. This approach is widely used in the literature, see, e.g., [1,5,7]. Here we will propose a different selection procedure, which may be easier to apply. Let $Q(\theta)$ be the observability matrix given by (11). Since $\operatorname{det} Q(\theta) \neq 0$ is required for observability, it is reasonable to expect that the observability hence synchronization properties become poorer as $Q(\theta)$ is closer to being singular. This property can be justified analytically by using singular values of $Q$; see, e.g., [11]. For a given eigenvalue set, by using $\Delta=Q A Q^{-1}$ and $\hat{C}=C Q^{-1}=(10, \ldots, 0)$, one can easily find the required gain $\hat{K}$ such that the eigenvalues of $\Delta-\hat{K} \hat{C}$ are precisely the given set. Then the required gain is $K=Q^{-1} \hat{K}$; see [11]. Hence, as $Q$ is closer to being singular, larger gains will be required to stabilize $A-$ $K C$. Such larger gains will result in larger transients in the error dynamics, and this may result in the loss of synchronization. Motivated by this argument, as $\left\|Q^{-1}\right\|=\left[\sigma_{\min }(Q)\right]^{-1}$, where $\sigma_{\min }(Q)$ is the minimum singular value of $Q$, and $\|\cdot\|$ is the matrix norm induced by the standard Euclidean norm, we propose the following selection criterion for appropriate $\theta_{*}$ :

$$
\theta_{*}=\max _{\theta \in \Omega} \sigma_{\min }(Q(\theta)) .
$$

To illustrate the ideas presented above, consider the hyperchaotic Rössler system given by (7). Let us express (7) in the form of (1) with $u=\left(x_{1} \cdots x_{4}\right)^{T}, f(u)=A u+$ $o(u)$, where $A$ is the linear part and $o(u)=\left(\begin{array}{lll}0 & 0 & 3\end{array}+\right.$ $\left.x_{1} x_{3} 0\right)^{T}$. We will choose $s=C u$, where $C^{T} \in \mathbf{R}^{4}$ is a vector to be determined. First consider the case $s=x_{i}$, $i=1,2,3,4$, i.e., plain phase variables. In this case, $C^{T}$ is the $i$ th unit vector. A simple calculation shows that with $s=x_{1}$ or $s=x_{2}$, the observability condition holds and synchronization may be possible, whereas with $s=x_{3}$ or $s=x_{4}$, detectability condition does not hold, 
hence synchronization is not possible with a differentiable observer. In [5], a full order observer is proposed, and it was reported that synchronization is not observed when a plain phase variable is used as a synchronization signal. According to our results, this is justified for $s=x_{3}$ or $s=x_{4}$. In [7], a full and an expanded order observer are proposed and it was reported that for $s=x_{2}$, synchronization is possible. Below we will show that this is also possible for $s=x_{1}$. Note that for $s=x_{1}$, we have $\sigma_{\min }(Q)=0.2032$, whereas for $s=x_{2}$ we have $\sigma_{\min }(Q)=0.0008$; hence according to (13), $s=x_{1}$ is a better choice. In the simulations, we use the following full order observer proposed in $[8,9]$ :

$$
\dot{v}=A v+o(v)+K(s-C v),
$$

where $s=C u$, and $K$ is to be determined. For $s=x_{1}$,

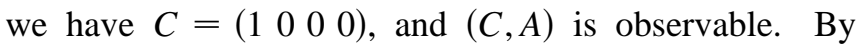
using $S=\{-1,-0.8,-0.6,-0.5\}$ as the eigenvalue set, we obtained $K=(3.2-3.51980 .4923$ $-1.4583)^{T}$ as the required gain. With these choices, we simulated (7) and (14) for $u(0)=\left(\begin{array}{llll}-20 & 0 & 0 & 15\end{array}\right)^{T}$ and $v(0)=0$, and the Euclidean norm of the resulting error $e=u-v$ is shown in Fig. 1 as dashed lines. We note that $u(0)$ is chosen according to [12]. Here $\|e(0)\|=25$, which is relatively large, yet synchronization is achieved in about 13 time units. We also searched various candidates for $C$ and for $C=\left(\begin{array}{llll}1 & 0 & 0 & -1\end{array}\right)^{T}$ we obtained $\sigma_{\min }(Q)=0.4597$, which is the largest value we found in our search. This indicates that $s=x_{1}-x_{4}$ may be a better choice than $s=x_{1}$. In this case $K=\left(\begin{array}{llll}2.4237 & -3.1102 & 0.243 & 0.7763\end{array}\right)^{T}$ leads to the same eigenvalue set $S$ for $A-K C$. By using these and the same $u(0), v(0)$ given above, we simulated (7) and (14), and the result is shown in Fig. 1 as a solid line. In this case, we observed a larger error in the transients, but synchronization is faster, i.e.,

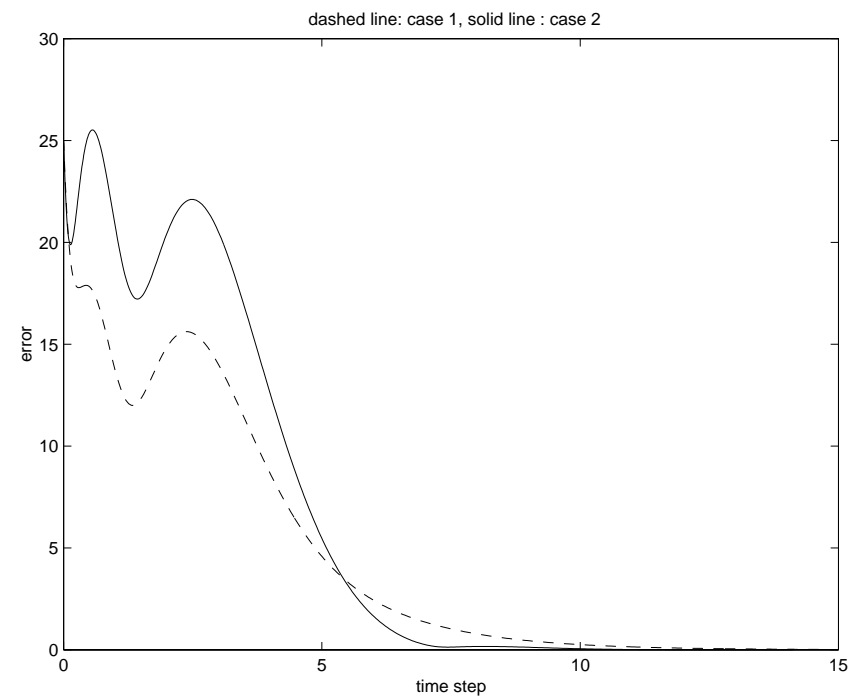

FIG. 1. Norm of synchronization errors for $s=x_{1}$ (dashed line) and for $s=x_{1}-x_{4}$ (solid line) for the hyperchaotic Rössler system (7). in about 9 time units. We also considered the case $C=(\sin \theta 0 \cos \theta 0)$, which is used in [5], and by using appropriate Lyapunov exponents $\theta=\pi / 3$ was reported as the best choice. By using (13), we obtained $\theta_{*}=0.54 \pi$, which resulted in $\sigma_{\min }(Q)=0.2068$. As compared with the case $s=x_{1}$, which has $\sigma_{\min }(Q)=0.2032$, we expect slight improvement. In this case, $K=\left(\begin{array}{llll}3.2873 & -3.5414 & 0.4899 & -1.5334\end{array}\right)^{T}$ leads to the same eigenvalue set. By using these and the same $u(0), v(0)$ as given above, simulation results are similar to that of case 1 in Fig. 1, with a slightly faster sychronization (in about 11 time units). Finally, we considered the case $C=(0 \cos \theta 0 \sin \theta)$ used in [7], and by using (13) we obtained $\theta_{*}=0.6 \pi$ as the best choice, which resulted in $\sigma_{\min }(Q)=0.1621$. In this case, $K=\left(\begin{array}{llll}-9.6911 & -4.1926 & -0.4888 & 2.0024\end{array}\right)^{T}$ leads to the same eigenvalue set. As can be seen, here gains are relatively larger than the ones obtained in previous cases, which is due to a relatively small $\sigma_{\min }$. In this case, in our simulations with the same $u(0)$ and $v(0)=0$ we could not observe synchronization. But with the same $u(0)$ and $v(0)=\left(\begin{array}{llll}-10 & 0 & 0 & 10\end{array}\right)^{T}$, synchronization is achieved (in about 12 time units). In this case we have $\|e(0)\|=11.18$, which is smaller than the cases considered previously, and apparently this is due to smaller $\sigma_{\min }$.

*Email address: morgul@ee.bilkent.edu.tr

[1] L. M. Pecora and T.L. Carroll, Phys. Rev. Lett. 64, 821 (1990).

[2] L. M. Pecora and T.L. Carroll, Phys. Rev. A 44, 2374 (1991).

[3] K. M. Cuomo and A. V. Oppenheim, Phys. Rev. Lett. 71, 65 (1993).

[4] L. Kocarev, K. S. Halle, K. Eckert, L. O. Chua, and U. Parlitz, Int. J. Bifurcation Chaos Appl. Sci. Eng. 2, 709 (1992).

[5] J. H. Peng, E. J. Ding, M. Ding, and W. Yang, Phys. Rev. Lett. 76, 904 (1996).

[6] G. Grassi and S. Mascolo, IEEE Trans. Circuits Syst. I, Fundam. Theory Appl. 44, 1011 (1997).

[7] C. K. Duan and S. S. Yang, Phys. Lett. A 229, 151 (1997).

[8] Ö. Morgül and E. Solak, Phys. Rev. E 54, 4803 (1996).

[9] Ö. Morgül and E. Solak, Int. J. Bifurcation Chaos Appl. Sci. Eng. 7, 1307 (1997).

[10] A. J. Krener, Systems and Networks: Mathematical Theory and Applications (Akademie Verlag, Berlin, 1994).

[11] C. T. Chen, Linear System Theory and Design (Holt, Rinehart, and Winston, New York, 1984).

[12] O. E. Rössler, Phys. Lett. 71A, 155 (1979).

[13] F. M. Callier and C. A. Desoer, Linear System Theory (Springer-Verlag, New York, 1991).

[14] X. Xia and W. Gao, Syst. Control Lett. 11, 319 (1988).

[15] X. Xia and M. Zeitz, Int. J. Control 66, 943 (1997).

[16] R. E. Amritkar and N. Gupte, Phys. Rev. E 47, 3889 (1993). 